\title{
BMJ Open Impact of a public health intervention for active surveillance and mitigation of SARS-CoV-2 in a district from Buenos Aires province, Argentina: a descriptive epidemiological study
}

\author{
Favio Crudo,, ${ }^{1,2}$ Mariana Fernández,, ${ }^{1,2}$ Marcelo Rodríguez Fermepín,, 3,4 \\ Andrea Carolina Entrocassi, ${ }^{3,4}$ Karina Angela Cardone, ${ }^{5}$ \\ Fernando Spina Markmann, ${ }^{5}$ Maria Victoria Periago (D) , ${ }^{1,6}$ Roberto Chuit, ${ }^{7}$ \\ Marcelo Claudio Abril ${ }^{1}$
}

To cite: Crudo F, Fernández M, Rodríguez Fermepín M, et al. Impact of a public health intervention for active surveillance and mitigation of SARS-CoV-2 in a district from Buenos Aires province, Argentina: a descriptive epidemiological study. BMJ Open 2021;11:e053595. doi:10.1136/ bmjopen-2021-053595

- Prepublication history and additional supplemental material for this paper are available online. To view these files, please visit the journal online (http://dx.doi.org/10.1136/ bmjopen-2021-053595).

Received 18 May 2021

Accepted 15 November 2021

Check for updates

(C) Author(s) (or their employer(s)) 2021. Re-use permitted under CC BY-NC. No commercial re-use. See rights and permissions. Published by BMJ.

For numbered affiliations see end of article.

Correspondence to Dr Maria Victoria Periago; vperiago@mundosano.org

\section{ABSTRACT}

Background The first case of SARS-CoV-2 was reported in Argentina on 3 March 2020. Measures to limit the spread of the virus were implemented, including complete lockdown (26 March). Nonetheless, the virus spread throughout the country, with a first peak of almost a million cases in October. On 30 November, the government's recommendation switched from social, preventive and compulsory isolation, to social, preventive and compulsory distancing.

Objectives To describe a tailored public health strategy to mitigate the spread of SARS-COV-2 and determine its behaviour in San Antonio de Areco district from Buenos Aires province (Argentina) through a private-public association.

Design, setting and participants Surveillance of the virus was performed with the local healthcare system, through early identification of cases and the systematic study of each infected individual and contact, regardless of symptomatology, using telemedicine and a COVID-19specific outpatient clinic. Real-time PCR was used for detection using both individual and pooled samples, with a 12-hour turnaround time.

Results Up to 30 November, a total of 2426 suspected cases were analysed and 578 were confirmed.

Surveillance of health personnel and at-risk populations proved effective, mitigating viral spread. Pooling samples allowed reduction of operator time, helped reduce costs, and allowed detection of both symptomatic and asymptomatic cases.

Conclusion After 8 months of protocol implementation, the strategy to intensively survey groups at higher epidemiological risk and the systematic search for asymptomatic cases with the incorporation of pooled PCR for diagnosis, in combination with individual testing, is an efficient and viable option in populations with similar characteristics, in the frame of social isolation.

\section{INTRODUCTION}

COVID-19 is a respiratory infectious disease caused by SARS-CoV-2. ${ }^{1}$ The virus has rapidly

\section{Strengths and limitations of this study}

- This study describes the positive impact of a public health strategy implemented in a district from the province of Buenos Aires (Argentina) during a large period of time, from the beginning of the epidemic in the country (March 2020), when a lockdown was put in place, to the end of the compulsory isolation phase in November 2020.

- The public health intervention was tailored for the district in a private-public partnership, using pooled nasopharyngeal swabs for surveillance and individual swabs from diagnosis, assuring rapid detection for both rapid isolation and early treatment, thus avoiding saturation of the health services.

- This is the first study, to our knowledge, conducted in the region of Latin America to assess the impact of such an intervention, showing a successful strategy to mitigate viral spread and reduce the number of deaths.

- This protocol with intensive surveillance and contact tracing worked well during lockdown in a small district of the province of Buenos Aires where public healthcare is available free of charge, and might not be extrapolated to localities with very large populations. Moreover, all the health and social care is under direct responsibility of the municipality, which facilitated planning and coordination of activities.

spread from Wuhan (China) to the rest of the world and as of 25 August 2021, there are 213050725 confirmed cases and 4448252 deaths in 236 countries, areas or territories. ${ }^{2}$ In Argentina, the first case was imported ${ }^{3}$ and notified on 3 March 2020 by the national surveillance system. ${ }^{4}$ As of 30 November 2020, the virus had spread to all 23 provinces and the country's capital city with a total of 1407277 cases and 38216 deaths. ${ }^{5}$ Initially, 
the most affected areas of the country were the capital city and surrounding areas located in Buenos Aires province; subsequently, the number of cases and deaths dramatically increased within the rest of the country.

Until an effective and accessible vaccine or treatment is available for the entire population, public health policies should include actions for the COVID-19 pandemic to avoid the increase of new cases and slow down the appearance of critical manifestations of the disease that overstretch the health system. Measures such as social distancing, personal hygiene and the use of face masks, on one side, and the identification of infected individuals on the other, are the leading public health measures to mitigate the spread of the disease. ${ }^{6-12}$ Additionally, diagnosis of presumptive cases must be performed in a systematic and timely manner to comply with the identification and subsequent isolation of infected individuals, thus also allowing rapid contact tracing. Although this would be the most effective strategy, there are specific challenges to its implementation due to asymptomatic, paucisymptomatic and pre-symptomatic carriers of the infection that are capable of maintaining the infection for several days (7-10 days). ${ }^{1}{ }^{12}$ Moreover, a prompt, specific and sensitive diagnosis requires the use of a PCR technique from nasopharyngeal swabs. This methodology is the most sensitive so far but requires specific reagents, specialised laboratories, trained personnel, time and a coordinated effort. According to each setting across the country, these elements may be more or less critical, depending on the available resources (technical, human and infrastructure) and opportunity. There are many public and private laboratories performing diagnosis using real-time polymerase chain reaction (RT-PCR) for viral detection, having different molecular targets and protocols. However, resources are limited, and the available laboratories performing PCR for SARS-CoV-2 detection are not distributed equally in the country, usually being concentrated in large capital cities and not in small districts.

In order to maximise detection and contact tracing capacities, different actions that combine effective epidemiological strategies with recently developed diagnostic tools that may be implemented and adapted to the different spatial-temporal scenarios are needed. The strategy presented herein was implemented in San Antonio de Areco (SAA) district, Buenos Aires province; and is based on a multistep strategy, including the systematic search for asymptomatic carriers through intensive epidemiological surveillance (health personnel, at-risk populations and exhaustive search of SARS-CoV-2 infection) and a systematic contact tracing of confirmed cases for their early isolation.

Due to the appearance of cases in the country and the confirmation of the first report of an imported case in SAA district on 22 March 2020, ${ }^{3}$ a public-private association was formed between two non-profit organisations, the municipality and a public academic institution to coordinate efforts to mitigate the spread of the disease. Herein, the temporal dynamics of SARS-CoV-2

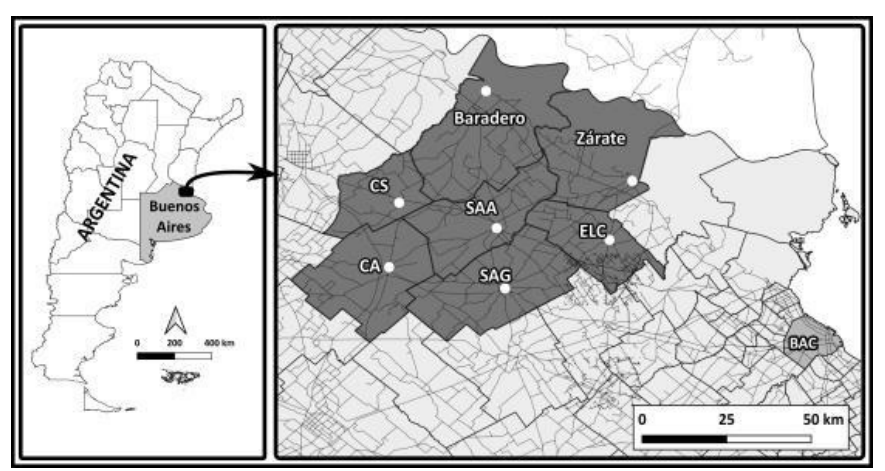

Figure 1 Map of the study area. On the left panel is Argentina with the province of Buenos Aires shaded. On the right panel is a close-up of the province, showing the district of San Antonio de Areco (SAA) and the surrounding districts of Baradero, Zárate, Exaltación de la Cruz (ELC), San Andrés de Giles (SAG), Carmen de Areco (CA) and Capitán Sarmiento (CS). Shaded in light grey, situated $113 \mathrm{~km}$ to the southeast, is Buenos Aires City (BAC). This map was elaborated using QGIS V.3.4 ${ }^{30}$ and layers from the Instituto Geográfico Militar. ${ }^{31}$

transmission in SAA, as well as the clinical and epidemiological characteristics of those who developed COVID-19 through the implementation of a proactive public health protocol, are described. In addition, the public health approach is presented together with the results of up to 30 November 2020 when the recommendations of the Argentinian government switched from social, preventive and compulsory isolation (SPCI) or lockdown, ${ }^{13}$ to social, preventive and compulsory distancing ${ }^{14}$ where certain non-essential activities were permitted although schools remained closed and more than 10 individuals could not gather in either public or private spaces.

\section{METHODS}

\section{Study setting and location}

The district of SAA is located in the northeast region of Buenos Aires province, approximately $113 \mathrm{~km}$ from the city of Buenos Aires (figure 1), and with a population of 25251 inhabitants. ${ }^{15}$ Since the district heavily relies on tourism and the agro-livestock sector for its income, it has a daily interchange of people and products from Buenos Aires City and the surrounding metropolitan area.

The public health system in SAA is organised around a municipal Health Secretariat and consists of the Municipal Hospital 'Emilio Zerboni' and a network of six centres of primary healthcare. The hospital is the only institution with hospitalisation capacity of a total of 60 beds, four of which are intensive care units (ICUs) with five ventilators. Additionally, SAA has a municipal residence for the elderly and two private institutions for geriatric care, and one institution for disabled individuals. Buenos Aires province has its own Ministry of Health, located in the capital of the province, La Plata (around $180 \mathrm{~km}$ away), and the province itself is divided into 12 sanitary regions. SAA is located in Sanitary Region IV, together 
with 12 other districts, and the health coordination for this region is located in Pergamino (150 km away), where the main reference hospital is located.

\section{Study design}

The protocol consisted of the implementation of public health actions following national norms and guidelines in the context of the COVID-19 pandemic through the use of quantitative data analysis. Basic parameters gathered included: cartography, size and population pyramid, proportion of the population defined as high risk for COVID-19, available healthcare services, number of healthcare workers, and number and characteristics of industrial factories defined by epidemiological transmission risk (ie, chicken farms, meat packing plants and others).

\section{Public-private association}

This project was elaborated after the first case of COVID-19 was detected in Argentina in order to model an epidemiological approach for the development of new and effective strategies to mitigate the spread of the virus and to study its behaviour in a small locality of the country where the health system could rapidly respond. For this purpose, and to be able to have all the resources necessary to implement the protocol, two non-governmental associations which have worked together in the past specifically in the field of neglected tropical diseases, ${ }^{16}$ Fundación Mundo Sano (FMS) and Asociación para el Desarrollo Sanitario Regional (ADESAR), engaged the local public health sector through the municipality of SAA and the Universidad de Buenos Aires. An agreement between all four institutions was signed. The diagnostic laboratory collaborating in this study, the Clinical Virology Laboratory, belongs to the Hospital de Clínicas 'José de San Martín', a hospital from the Universidad de Buenos Aires, located in Buenos Aires City.

\section{Preparedness and contingency planning in response to COVID-19}

In preparation for a response to the appearance of COVID-19 cases, the public health system improved its structure and services by increasing the total number of hospital beds to 80 , duplicating both the number of ICU beds and the number of ventilators. A new area of intensive care was set up specifically for patients with COVID19. Moreover, an area for isolation of hospitalised patients with mild COVID-19 was set up in the hospital and a hotel was conditioned for the isolation of asymptomatic individuals and those with mild symptoms. A module for the health personnel to use as a dressing room and decontamination was set up with a dirty and clean area, and a neighbouring school was conditioned for administration personnel of the hospital and outpatient services.

Additionally, all personnel were trained for this new situation and clinical management was reformulated so as to minimise exposure with presumptive patients. Therefore, a team for evaluation and treatment was set up and tools and supplies were incorporated, such as the digitisation of images and different therapeutic and diagnostic options. Another team was set up for remote follow-up of mild and asymptomatic patients and contact tracing.

\section{Target population and case identification}

All the inhabitants of the district of SAA, regardless of sex or age, were included. Those individuals who did not reside in the district were excluded unless they were individuals living outside SAA but working in the district. Identification of cases was performed in a multistep strategy using telemedicine, beginning with a remote medical attention system composed of a phone line available exclusively for individuals with respiratory symptoms. This system was coordinated by a medical team to evaluate the need for a clinical evaluation at the hospital and a real-time RT-PCR test. A COVID-19-specific hotline was set up for SAA; all calls were received by specially trained personnel and an epidemiological questionnaire (online supplemental data: Epidemiological Questionnaire) was used to determine if the individual fitted the definition of a suspect case. If the case definition was met, appointments were given in less than 24 hours. A specific range of time and a COVID-19-specific entrance were set up in the hospital for sampling collection. A specially trained nurse with appropriate personal protective equipment (PPE) would take the nasopharyngeal samples using a specimen collection cabinet to avoid infection as per Centers for Disease Prevention and Control (CDC) guidelines. ${ }^{17}$

All confirmed cases were placed under the following epidemiological classifications: close contact of a confirmed SARS-CoV-2-positive individual, healthcare worker taking into consideration the place of work of the worker, travellers who went to areas with confirmed community transmission within the country, individuals who work as security personnel (military personnel, police force, private security), an imported case (a person who came from another country), or/and unlinked case (the origin of the infection is unknown). If an individual fitted into more than one of these categories, the case was classified using the epidemiological risk categorisation (ie, even if a healthcare worker was a close contact of a confirmed case, they were classified as a healthcare worker).

\section{Population under epidemiological surveillance}

The entire population from SAA was under surveillance. Case and contact definitions are described in online supplemental table 1 and followed national guidelines, ${ }^{18}$ although in some cases the definitions adopted by the study were more sensitive. Specific at-risk populations (online supplemental table 2) including healthcare workers, personnel from closed institutions, patients undergoing surgeries, workers under risk due to their occupation, symptomatic compatible cases not included in the definition of a suspected case, and asymptomatic contacts of a confirmed case were placed under routine epidemiological surveillance based on a risk scale in 
order to achieve rapid detection and isolation of infected individuals.

The risk scale used was as follows: (1) high-risk individuals-those who, due to their work activities, may act as sources of transmission, such as active healthcare workers from the hospital, active healthcare workers from primary healthcare posts, workers from residential homes for elderly or disabled individuals, and individuals undergoing planned surgery. These individuals were tested in pools every 15 days. Among healthcare workers, those who worked in more than one health institution were considered to be at higher risk, especially those who worked in institutions from districts where community viral transmission was occurring. In closed institutions (like residencies for the elderly), workers who worked in more than one institution or lived with a person who worked in a district with community viral transmission were also considered to be at a higher risk. Those symptomatic compatible cases that remained in areas with community viral transmission for a specific time or in contact with an individual from such areas without PPE were also considered higher risk individuals; these individuals were tested in pools every week. (2) Medium-risk individuals-those who, due to their work activities, had the possibility of acting as sources of transmission given their contact with a large number of different individuals, such as security workers or commercial workers from produce/food shops or other essential elements of daily use, as well as workers from industrial plants. (3) Incidental-risk individuals-those symptomatic cases that do not fit the definition of a suspected case but have occupations considered as risky, such as workers from industrial plants. In those cases, an individual risk score was elaborated for each individual to weekly survey $10 \%$ of asymptomatic workers. These individuals were selected and evaluated on a weekly manner based on epidemiological interviews to select those who would be more at risk, depending on where they lived and with whom, as well as the presence of symptoms in household contacts. Moreover, incidental risk in a population was taken into account, such as a group of individuals that, for some incidental reason, were at higher risk of exposure in a particular place for a certain period and where identification of a specific contact could not be determined.

\section{Patient and public involvement}

The epidemiological approach described within and applied in SAA was designed as a public-private association between the municipality, the Health Secretariat, a public academic institution and two non-governmental organisations. As such, the well-being of all the inhabitants of SAA was taken into consideration as a public health measure, with regular communication from the municipality and the Health Secretariat to inform the measures that would be taking place through the different phases of the pandemic.

\section{Diagnosis of SARS-CoV-2}

Nasal and pharyngeal swabs were taken by healthcare personnel and placed in the same tube containing $2 \mathrm{~mm}$ of saline solution. Samples were kept at $2^{\circ} \mathrm{C}-8^{\circ} \mathrm{C}$ and delivered within 4 hours for SARS-CoV-2 detection to the Clinical Virology Laboratory at the University Hospital, where the samples were immediately processed. Nucleic acid extraction was made using a commercial semiautomated viral RNA extraction kit (King Fisher Duo, Thermo Scientific), according to the manufacturer's instructions. SARS-CoV-2 was detected by real-time PCR according to the CDC protocol, ${ }^{19}$ targeting N1, N2 viral genes and human RNAse $\mathrm{P}$ in individual wells, using FAM-BHQ-1labelled probes, on an Abi 7500 real-time thermocycler (Applied Biosystems). Samples were considered positive for SARS-CoV-2 if both N1 and N2 targets were amplified at any CT values less than 40. If only one target was detected, the whole extraction and amplification process was repeated. If the discordance between target amplification persisted, a new sample was collected. Negative samples with no amplification of RNAse P were considered invalid.

Only laboratory-confirmed cases are included herein for the consistency of case definition throughout the 8 months of the epidemic in SAA and for comparison; data from the rest of the country and the world were updated to the same point in time.

\section{Epidemiological surveillance through sample pooling}

For SARS-CoV-2 surveillance, pooled samples were used for detection by real-time RT-PCR from nasal and pharyngeal swabs with rapid turnaround time. At the time this protocol was designed, there was a lack of international guidelines available regarding simple pooling methods for SARS-CoV-2 detection. Therefore, a swab-pooling method was evaluated and validated at the Clinical Virology Laboratory from the University of Buenos Aires. Concretely, a single swab was used to collect a sample for the nose and pharynx and it was placed in the same tube together with the swabs from four other individuals, thus comprising a pool of five individuals, which proved to be cost-benefit efficient when COVID-19 incidence was lower than $5 \%$. If the five-sample pool turned out to be negative, all the individuals were considered negative. If the five-sample pool was positive, then individual nasopharyngeal swabs were taken to determine which of the five individuals was infected. All results were available within 12 hours.

\section{Contact tracing and isolation}

All symptomatic individuals were isolated until infection by SARS-CoV-2 was verified. If SARS-CoV-2 was undetected, individuals had to remain isolated until they no longer had symptoms. For every positive case confirmed by real-time RT-PCR, isolation for 10 days since the onset of symptoms was assured through daily telephone calls by the health team and social services. Social services would determine the need to give assistance for grocery shopping 
or other specific needs based on the socioeconomic situation of the affected individuals. All symptomatic individuals with a positive test would then be further questioned over the phone in order to determine transmission chains and isolate all close contacts who had been in contact with the primary case 48 hours prior to the onset of symptoms. The questionnaire was also used to detect all the contacts of the primary case, taking into consideration all those individuals who had been in contact 7-10 days prior to the onset of symptoms. All close contacts/co-inhabitants of the primary case were isolated and tested using individual real-time RT-PCR. All those incidental contacts who had been in contact with the primary case 48 hours prior to the onset of symptoms were tested 7-10 days after onset of symptoms using pooled real-time RT-PCR. Through this contact tracing actions, most symptomatic individuals and their contacts were captured by the system which was complemented by a monitoring app for symptomatic individuals. As the epidemic progressed, volunteers were recruited to assure rigorous contact tracing. Confirmed cases of COVID-19, either symptomatic or subclinical, as well as their contacts, were monitored on a daily basis, by trained personnel through the app 'Areco Symptoms'. This app was developed specifically for the project by Sigmind ${ }^{20}$ according to the healthcare team's objectives. Trained individuals coordinated actions with social workers and generated contact with the medical team if symptoms worsened over time in those individuals with no comorbidities or if symptoms persisted for more than 5-6 days in those individuals with comorbidities. In those cases, an appointment was given for clinical evaluation in the COVID-19-specific outpatient clinic that was set up next to the hospital. Patients who did not require hospitalisation and had cleared the infection were discharged, and thus monitoring was finalised.

\section{Data analysis and case reports}

Quantitative data were consolidated weekly and analysed to determine the number of individuals studied by realtime RT-PCR (both individually and pooled), the number of confirmed cases, the number of deceased individuals, the number of confirmed cases and their relatedness, the proportion of symptomatic/asymptomatic, general hospitalisations, intensive care hospitalisations, and number and average attack rates by case. As the weekly report of the epidemiological situation was elaborated, establishment and modification of measures were suggested to the municipal authorities.

Cases were classified into asymptomatic and symptomatic based on their clinical presentation. Asymptomatic individuals were those with a SARS-CoV-2-positive realtime RT-PCR but with no symptoms compatible with COVID-19. Symptomatic individuals were classified into either mild or moderate symptoms, severe symptoms or critical symptoms based on the guidelines of the National Institutes of Health of the USA. ${ }^{21}$

The descriptive analysis was performed using morbidity and mortality indicators for the entire period of the study,

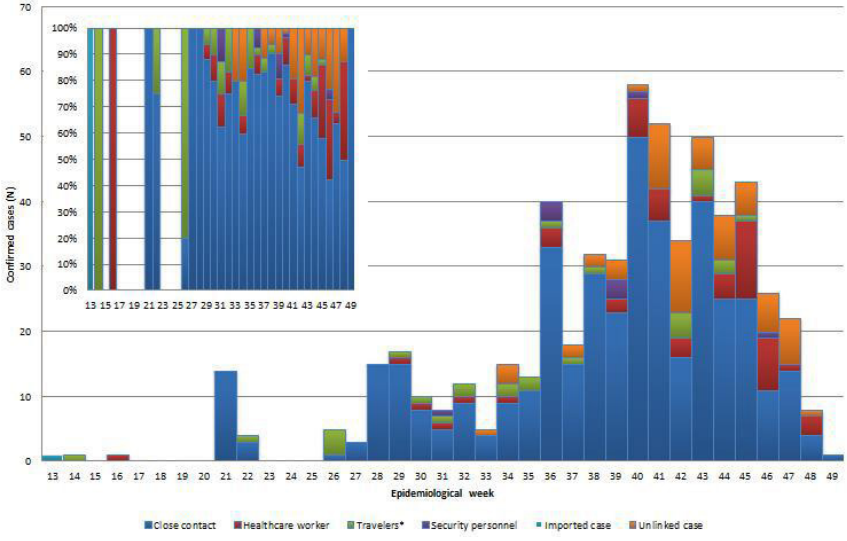

Figure 2 Epidemiological curve of weekly confirmed COVID-19 cases according to epidemiological classification and proportion. Epidemiological week (EW) 13 to EW49, 2020; San Antonio de Areco, Buenos Aires, Argentina. *Travellers to an area with confirmed community transmission.

spanning the first 8 months. Rates were calculated using national projections for 2020 from the Instituto Nacional de Estadística y Censos (National Institute for Statistics and Census) ${ }^{15}$ as denominators. Qualitative variables are expressed as absolute and relative numbers. Rates and proportions were calculated with a 95\% CI. Quantitative variables are expressed as measures of central tendency and dispersion. R (V.4.0.4) was used for statistical analyses.

\section{Role of the funding source}

Additional costs for the project are funded by FMS, while ADESAR, Universidad de Buenos Aires and the Health Secretariat of SAA provide their structure and personnel. Funders did not have a role in the study's design or collection, analysis, and interpretation of data nor in the writing.

\section{RESULTS}

\section{Description of cases}

During the first 49 epidemiological weeks (EWs) of the epidemic in SAA, from 22 March to 30 November, a total of 2426 suspected cases were analysed through nasopharyngeal swabs and real-time RT-PCR; 578 cases were confirmed (23.8\%; $95 \%$ CI $22.1 \%$ to $25.6 \%$ ) and 1848 were discarded. Figure 2 shows the epidemiological curve of weekly confirmed COVID-19 cases according to the epidemiological classification: close contact, healthcare worker, travellers to areas with confirmed community transmission, security personnel, imported case or unlinked case. The number of unlinked cases seems to increase during EW41 but overall, the proportion of unlinked cases was low $(\mathrm{n}=63 ; 10.9 \% ; 95 \%$ CI $8.5 \%$ to $13.8 \%)$. Of the 578 confirmed cases, 420 (72.7\%; $95 \%$ CI $68.8 \%$ to $76.2 \%)$ were close contacts, $56(9.7 \%$; $95 \%$ CI $7.5 \%$ to $12.5 \%)$ were health personnel, 29 (5.0\%; $95 \%$ CI $3.4 \%$ to $7.2 \%$ ) were travellers to areas with confirmed community transmission, $9(1.6 \%$; $95 \%$ CI $0.8 \%$ to $3.0 \%)$ 


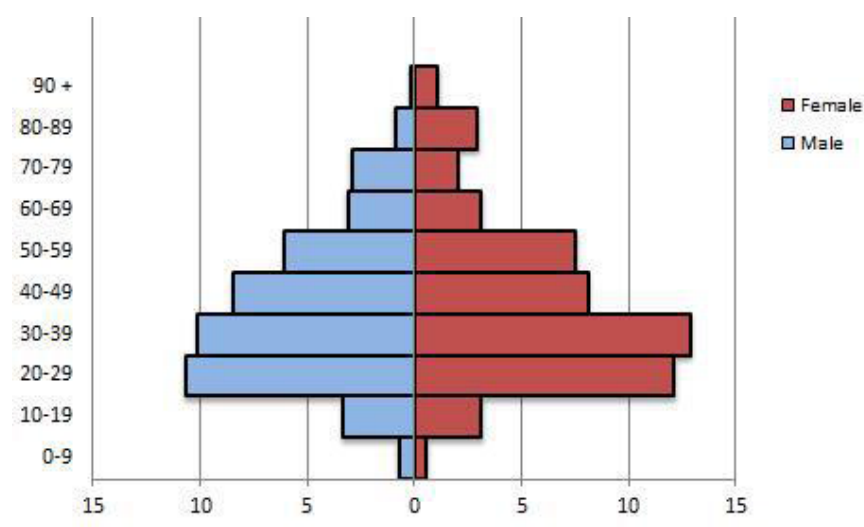

Figure 3 Per cent distribution of confirmed cases according to gender and age group, epidemiological week (EW) 13 to EW49, 2020; San Antonio de Areco, Buenos Aires, Argentina.

were security personnel and only $1(0.2 \%$; $95 \%$ CI $0.0 \%$ to $1.1 \%$ ) was an imported case. Among the confirmed cases, 10 passed away, representing an accumulated case fatality rate (CFR) of $1.7 \%$ (95\% CI $0.9 \%$ to $3.3 \%$ ) and a specific mortality rate of 4 in 10000 inhabitants $(95 \%$ CI 2.0 to $7.5)$. Deceased individuals were all older than 65 years of age (range between 66 and 96 years) with comorbidities such as cancer, neurological disease, cerebral vascular accidents or chronic lung disease; 6 of the 10 deceased were women.

Regarding the age and sex distribution of the 578 confirmed cases, $302(52.2 \%)$ were female and 276 $(47.8 \%)$ male, with a median age of 38 years, a range between 2 and 96 years, and an IQR of 28-53 (figure 3). Age distribution shows that $249(45.8 \%)$ of the patients were between 20 and 39 years of age (missing age from 34 individuals).

Approximately one-third of the confirmed cases were asymptomatic ( $\mathrm{n}=167 ; 29.7 \%$; $95 \%$ CI $26.0 \%$ to $33.7 \%)$, 59 (10.5\%; $95 \%$ CI $18.1 \%$ to $13.4 \%$ ) had moderate symptoms who required admission or outpatient care, and only $11(2.0 \% ; 95 \%$ CI $1.0 \%$ to $3.6 \%)$ had severe and 12 (2.1\%; $95 \%$ CI $1.2 \%$ to $3.8 \%$ ) had critical symptoms. More than half of the confirmed cases had only slight symptoms ( $\mathrm{n}=314 ; 55.8 \%$; $95 \%$ CI $51.6 \%$ to $59.9 \%)$, missing data from 15 individuals. Only 82 (14.6\%; $95 \%$ CI $11.8 \%$ to $17.8 \%$ ) patients required hospitalisation, of which 4 required admittance to the ICU of the local SAA hospital and 3 of these required mechanical respiration. The average occupancy of ICU beds and requirement of mechanical respiration was 6 days. The median age of hospitalised patients was 58 years, with a range between 22 and 96 years and an IQR of 48-72; there are missing data from two individuals. Of the total number of hospitalised patients, $56.1 \%(\mathrm{n}=46)$ were women and $43.9 \%$ $(\mathrm{n}=36)$ were male.

The epidemiological situation of the Sanitary Region SAA that belongs to Sanitary Region IV confirmed 19040 cases and 567 deaths with a CFR of $3.0 \%(95 \%$ CI $2.7 \%$ to $3.2 \%$ ) during the first full 49 weeks of the epidemic. The case incidence rate per 1000 inhabitants for the region was 31.4 (95\% CI 31.0 to 31.9) while that of SAA was 22.9 cases per 1000 (95\% CI 21.1 to 24.8$).^{22}$ Moreover, the CFR for SAA is the lowest in the region (table 1).

\section{Surveillance of at-risk populations}

Surveillance of at-risk populations, classified as such based on their exposure, as detailed in online supplemental table 2, began on 14 April 2020. Since nasopharyngeal samples were taken to determine the presence of SARS-CoV-2 infection either in a weekly manner or every 15 days, depending on the risk of exposure, the total number of samples analysed, including cases and population under surveillance, was 8185; this corresponds to a test rate of 324 for every 1000 inhabitants $(95 \%$ CI 318.4 to 330.0). Of this 8185 samples, 5759 (70.4\%; 95\% CI $69.4 \%$ to $71.3 \%$ ) belonged to high-risk groups that were under weekly surveillance and the remaining 2426 (29.6\%; $95 \%$ CI $28.7 \%$ to $30.6 \%$ ) were contacts and symptomatic patients. Of these high-risk groups, 4489 (77.9\%; $95 \%$ CI $76.8 \%$ to $79.0 \%$ ) belonged to healthcare personnel (including the personnel from the municipal residence for the elderly).

The accumulated test positivity rate was $7.0 \%$ (95\% CI $6.5 \%$ to $7.6 \%$ ), except for the first two EWs, when only suspected individuals were tested. In the following EW, the test positivity rate was maintained below $10 \%$. On EW36, the test positivity rate started to increase, reaching a maximum peak of $17 \%$ on EW41, finally decreasing to $2 \%$ in the last week of November (figure 4).

Within the surveillance of healthcare personnel $(\mathrm{n}=358), 56$ positive cases were found $(15.6 \% ; 95 \%$ CI $12.1 \%$ to $19.9 \%$ ); 17 of them identified through active surveillance while the rest were symptomatic. Given the rapid detection of infected personnel, regardless of symptomatology, no other infected healthcare workers were detected. On the week of 13 July, surveillance of a

Table 1 Comparison of the COVID-19 epidemiological situation of Sanitary Region IV of the province of Buenos Aires (Argentina), as of 30 November 2020 describing the number of confirmed cases, deaths, cases per 1000 inhabitants and CFR

\begin{tabular}{lccccc}
\hline District & Population & Confirmed cases & Deaths & Cases per 1000 (95\% CI) & CFR (\%) (95\% CI) \\
\hline San Antonio de Areco & 25251 & 578 & 10 & $22.9(21.1$ to 24.8) & $1.7(0.9$ to 3.3) \\
Sanitary Region IV* & 605585 & 19040 & 567 & $31.4(31.0$ to 31.9) & $3.0(2.7$ to 3.2) \\
\hline
\end{tabular}

*Districts included in Sanitary Region IV: Arrecifes, Baradero, Capitán Sarmiento, Carmen de Areco, Colón, Pergamino, Ramallo, Rojas, Salto, San Andrés de Giles, San Antonio de Areco, San Nicolás and San Pedro.

CFR, case fatality rate. 


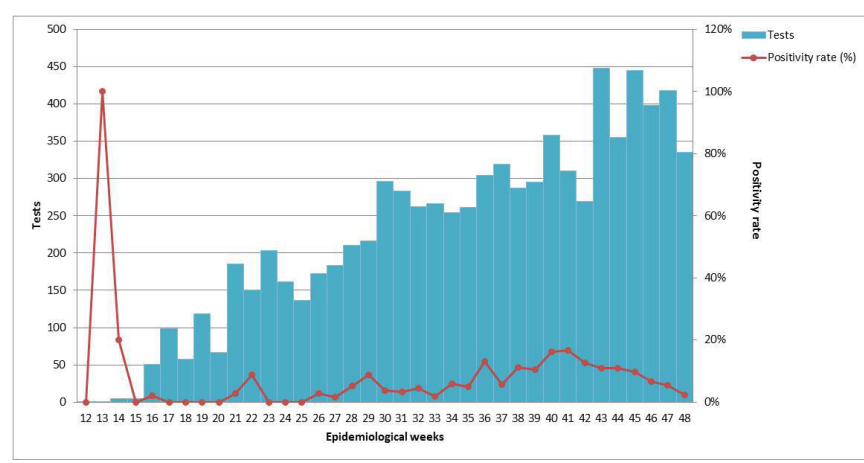

Figure 4 Diagnostic tests performed and test positivity rate in San Antonio de Areco, Buenos Aires, Argentina, from epidemiological week (EW) 13 to EW49, 2020.

private meat packing plant was implemented. A total of 240 samples (20 per week) were processed; and on EW34, two cases were confirmed, one resident from SAA and one resident from Pilar. A second poultry plant located in nearby Villa Lía, which is part of the district of SAA, has also been surveyed and a total of 132 samples have been processed. Three individuals were identified as positive for SARS-CoV-2, one resident from Villa Lía, one resident of San Andres de Giles and the other resident of Baradero. Contact tracing showed they had not infected other individuals. A third company-an agricultural machinery seller-incorporated surveillance of its workers. A total of 80 samples were processed and none of them were found to be positive.

\section{DISCUSSION}

In Argentina, a total of 1407277 cases and 38216 deaths from COVID-19 were confirmed as of 30 November, with a CFR of $2.7 \% .^{5}$ This was during the phase of the pandemic when lockdown, or SPCI, was mandated at the national level. During this lockdown phase, individuals had to work from home and could leave the house for essential shopping needs within their neighbourhood, travel was prohibited, schools were closed, all public cultural, religion and sporting events were cancelled, and non-essential stores and shopping centres were closed. Health and military/security personnel, migration, fire department, meteorology officials and air traffic control personnel were considered essential and were exempted, as well as other areas of the government and those who needed to care for someone with a disability or had an unforeseen emergency event, among others. ${ }^{13}$

In SAA, the total number of cases for the same period was 578 and 10 deaths from COVID-19, with a CFR of $1.7 \%$. Due to the different characteristics within Argentina, comparisons are often challenging. However, if SAA is compared with districts from the same Sanitary Region, in time, we would be able to determine if the implementation of this protocol has helped mitigate the spread of the virus and prevent deaths. The number of positive per 1000 inhabitants in SAA was 23 as of 30 November, below the average for the Sanitary Region for that same period, and the CFR was the lowest for all 13 localities comprising the region.

With respect to the characteristics of the confirmed cases, many previous studies have focused on hospitalised cases or have recorded small periods of time during the mitigation phase of the pandemic, spanning a few months. ${ }^{723-25}$ As in SAA, all ages were susceptible to infection, although most cases were in adults and there was no significant difference in gender. ${ }^{6}{ }^{23-25}$ With respect to the clinical presentation, as in previous studies, most individuals had mild infections and only a few required hospitalisation, with most moderate infections being treated as outpatients and only very few critical cases with hospitalisation in the ICU. ${ }^{6723-25}$ With respect to the characteristics of the deceased, in SAA most of them were older individuals with comorbidities as reported in other countries. $^{6} 723-25$

One of the endpoints of this study was to evaluate the possibility of incorporating population-based methods and epidemiological tools to reduce access barriers, perform rapid detection of cases and their isolation, and achieve mitigation of transmission of SARS-CoV-2 in the context of the pandemic and during a time when neither specific treatment nor vaccines were available. The focus on the disease's epidemiological aspects, and not on its clinical manifestations, has allowed early detection of asymptomatic carriers; therefore, limiting the spread of the virus as much as possible, with active testing, isolation and tracing, proved effective in other countries as well. $^{671112}$ In this study, the proportion of asymptomatic individuals with a confirmed real-time RT-PCR test was $29.7 \%$, which coincides with findings from other studies which also conducted rigorous contact tracing. ${ }^{26}$ The impact of the epidemiological surveillance of healthcare personnel during this mitigation phase was also effective. Given the rapid detection of infected personnel, regardless of symptomatology, $15.6 \%$ of all health workers were detected and isolated, limiting the spread of the virus to other workers or patients. In other studies, healthcare personnel were found to have high prevalence of infection. 6 7 23-25

The weekly surveillance of populations with incidental risk was also effective, given that during the first 2 months of implementation of this surveillance protocol, a cluster of 18 cases was promptly detected. These cases originated from a single worker from a store who obtained fruits and vegetables from a wholesaler. A similar situation was reported from neighbouring districts; therefore, a questionnaire was performed in all the fruit and vegetable stores from SAA (all of them obtain produce from wholesalers). A total of 13 individuals were tested every 10 days for a month and 4 of the individuals tested positive (confirmed on 24 June). All the contacts of these individuals were tested and only one resulted positive.

At the time of this protocol design, March 2020, there were few options for SARS-CoV-2 RNA detection, international guidelines were being developed and there were no specific studies on sample pooling. Pooling the 
samples by five, instead of pooling the extracted RNA only for real-time RT-PCR purposes, allowed the reduction of operator time and helped save extraction reagents and consumables for RNA extraction. It also proved to be a successful strategy for surveillance and detection of cases in the low-risk population and in contact tracing, since the results are processed rapidly and available after only 12 hours.

As the pandemic progressed, a number of studies supporting the sample choice of the protocol started being published, confirming that pooling nasal and throat swabs gave the highest sensitivity of detection, ${ }^{27}$ in comparison with nasopharyngeal swabs as the gold standard. Also, as of June 2021, specific CDC interim guidelines for pooling ${ }^{28}$ recommended the use of pooling by five to reduce the number of tests needed when the incidence of COVID-19 was lower than 5\%. Finally, most of the nucleic acid amplification tests for SARS-CoV-2 detection cleared by the US Food and Drug Administration proved to have a better performance when using swab pooling over transport media pooling, in agreement with our pooling design. ${ }^{29}$ During the development of this protocol, this sample pooling strategy should be reassessed periodically according to the development of the COVID-19 epidemic in SAA, its neighbour cities and the entire province of Buenos Aires.

This private-public partnership between two nongovernmental institutions, a municipality and a public university created the conditions needed to optimise resources and shorten the detection time of infected individuals regardless of their symptomatology, thus contributing to an effective cost-benefit in the implementation of the protocol. Moreover, the organisation and implementation of this epidemiological approach were feasible due to the political will of the municipality of SAA, where the hospital and all the healthcare and social services depend directly on the municipality and are free of charge. The extra resources needed to be able to test and rapidly obtain results for contact tracing, as well as to implement active surveillance, were accomplished due to the public-private partnership. Also, many private companies independently applied the active surveillance protocol, under the advice of the Health Secretariat, in order to avoid having to close.

This protocol was implemented in the context of a low national availability of laboratories with the necessary infrastructure for the detection of SARS-CoV-2, which is saturated due to the increasing epidemic. The SAA town authorities have manifested their concern for the disruption to the access to healthcare services during the pandemic, for which the implementation of interventions based on the use of appropriate technology constitutes a priority in health management. Implementing this surveillance protocol allowed the conformation of trained teams that can make the implementation sustainable by systematising procedures and designing tools that allow monitoring indicators of processes and results.
During the mitigation phase of the pandemic when specific treatment and vaccines were not available, the objective of the Health Secretariat and the municipality of SAA was clear: to reduce the transmission of the virus to avoid deaths. Some limitations to this approach are that it is not viable in the context of community transmission and it was applied in a municipality which controls all aspects of the healthcare and social services, avoiding bureaucracy between different government levels. Moreover, SAA is a small municipality which made it easier to cover the entire population; therefore, although public healthcare is free in the country, the rapid detection of cases required extra resources that may not be widely available.

In conclusion, after 8 months of protocol implementation, the strategy to intensively survey groups at higher epidemiological risk and the systematic search for asymptomatic cases via contact tracing, together with the incorporation of pooled PCR for surveillance and individual testing for diagnosis, both with a rapid turnaround time, proved to be an efficient and viable option that can be extrapolated to other populations with similar characteristics, in the frame of the mitigation phase of this global pandemic.

\section{Author affiliations}

${ }^{1}$ Mundo Sano Foundation, Buenos Aires, Argentina

${ }^{2}$ Association for Regional Health Development (ADESAR), San Antonio de Areco, Buenos Aires, Argentina

${ }^{3}$ Faculty of Pharmacy and Biochemistry, Chair of Clinical Microbiology/INFIBIOC Buenos Aires University, Buenos Aires, Argentina

${ }^{4}$ Hospital de Clínicas 'José de San Martín', Laboratory of Immunology and Clinical Virology, Buenos Aires University, Buenos Aires, Argentina

${ }^{5}$ Health Secretariat of San Antonio de Areco, San Antonio de Areco, Buenos Aires, Argentina

${ }^{6}$ National Council for Scientific and Technical Research, Buenos Aires, Argentina ${ }^{7}$ Institute of Epidemiological Research, National Academy of Medicine, Buenos Aires, Argentina

Acknowledgements We would like to thank all the health professionals and workers from the Association (Cooperadora) of the Municipal Hospital 'Emilio Zerboni' for their effort and collaboration, as well as all the volunteers who helped perform contact tracing. We would also like to thank all the laboratory team members from the Universidad de Buenos Aires who were involved: Dr Maria Lucía Gallo Vaulet; the biochemists Laura Alejandra Peker, Analía Patricia Toledano, David Ariel Sosa, Javier García and María H Irigoyen; the technician Esteban Bertani Marin; as well as Rodrigo Bruselario and Danila I Allegrini. We would like to acknowledge the valuable input from Ana Pereiro (Mundo Sano) and Alejandro Krolewiecki (Instituto de Investigaciones de Enfermedades Tropicales, Facultad de Ciencias de la Salud, Universidad Nacional de Salta). We would also like to thank Manuel 0 Espinosa (Mundo Sano) for the elaboration of figure 1.

Contributors FC is the principal investigator of the study described in this article and guarantor of the overall content. FC, MF, MRF, RC and MCA developed the original study protocols. FSM is the Secretary of Health of SAA. MCA was responsible for the agreement with the Universidad de Buenos Aires and contacting Sigmind. FC, MF and KAC conducted the study in SAA, while MRF performed the laboratory procedures. KAC, ACE and MVP gathered the data and wrote the first draft of the manuscript. MVP performed the final edits. All authors read and approved the final manuscript.

Funding This work was supported by private-public collaboration between Fundación Mundo Sano, Asociación para el Desarrollo Sanitario Regional (ADESAR), Universidad de Buenos Aires and the Health Secretariat of San Antonio de Areco. Award/grant number is not applicable. 
Map disclaimer The depiction of boundaries on the map(s) in this article does not imply the expression of any opinion whatsoever on the part of BMJ (or any member of its group) concerning the legal status of any country, territory, jurisdiction or area or of its authorities. The map(s) are provided without any warranty of any kind, either express or implied.

Competing interests None declared.

Patient consent for publication Not required.

Ethics approval Ethical approval was obtained from the Instituto de la Academica Nacional de Medicina, Buenos Aires, Argentina (reference number 36/20/CEIANM). The committee waived the need of a consent form given this is a study protocol applied by the Health Secretariat of San Antonio de Areco as a public health intervention to control the spread of SARS-CoV-2 in the population in the context of a global pandemic.

Provenance and peer review Not commissioned; externally peer reviewed.

Data availability statement Data are available upon reasonable request. All data relevant to the study are included in the article or uploaded as supplemental information. All study data were extracted from the national database, Sistema Nacional de Vigilancia en Salud-SNVS V.2.0, and can be obtained for research purposes by contacting epidemiologia@areco.gob.ar and following the data request process.

Supplemental material This content has been supplied by the author(s). It has not been vetted by BMJ Publishing Group Limited (BMJ) and may not have been peer-reviewed. Any opinions or recommendations discussed are solely those of the author(s) and are not endorsed by BMJ. BMJ disclaims all liability and responsibility arising from any reliance placed on the content. Where the content includes any translated material, BMJ does not warrant the accuracy and reliability of the translations (including but not limited to local regulations, clinical guidelines, terminology, drug names and drug dosages), and is not responsible for any error and/or omissions arising from translation and adaptation or otherwise.

Open access This is an open access article distributed in accordance with the Creative Commons Attribution Non Commercial (CC BY-NC 4.0) license, which permits others to distribute, remix, adapt, build upon this work non-commercially, and license their derivative works on different terms, provided the original work is properly cited, appropriate credit is given, any changes made indicated, and the use is non-commercial. See: http://creativecommons.org/licenses/by-nc/4.0/.

\section{ORCID iD}

Maria Victoria Periago http://orcid.org/0000-0002-1470-5146

\section{REFERENCES}

1 Ciotti M, Angeletti S, Minieri M, et al. COVID-19 outbreak: an overview. Chemotherapy 2019;64:215-23.

2 WHO coronavirus disease (COVID-19) Dashboard. Available: https:// covid19.who.int/ [Accessed 25 Aug 2021].

3 Rearte A, Baldani AEM, Barbeira PB. Características epidemiológicas de Los primeros 116974 casos de COVID-19 en Argentina, 2020. Rev Argent Salud Publica 2020;12:e5.

4 Sistema Nacional de Vigilancia en Salud - SNVs 2.0. data from. Available: https://www.argentina.gob.ar/coronavirus/informesdiarios/sala-de-situacion [Accessed 14 Nov 2020].

5 Sistema Nacional de Vigilancia en Salud - SNVs 2.0. data from. Available: https://www.argentina.gob.ar/coronavirus/informesdiarios/sala-de-situacion [Accessed 30 Nov 2020].

6 Jefferies S, French N, Gilkison C, et al. COVID-19 in New Zealand and the impact of the National response: a descriptive epidemiological study. Lancet Public Health 2020;5:e612-23.

7 Wong J, Chaw L, Koh WC, et al. Epidemiological investigation of the first 135 COVID-19 cases in Brunei: implications for surveillance, control, and travel restrictions. Am J Trop Med Hyg 2020;103:1608-13.

8 Pan A, Liu L, Wang C, et al. Association of public health interventions with the epidemiology of the COVID-19 outbreak in Wuhan, China. JAMA 2020;323:1915-23.

9 Du Z, Pandey A, Bai Y, et al. Comparative cost-effectiveness of SARS-CoV-2 testing strategies in the USA: a modelling study. Lancet Public Health 2021;6:e184-91.
10 Koo JR, Cook AR, Park M, et al. Interventions to mitigate early spread of SARS-CoV-2 in Singapore: a modelling study. Lancet Infect Dis 2020;20:678-88.

11 Pinkas J, Jankowski M, Szumowski kukasz, et al. Public health interventions to mitigate early spread of SARS-CoV-2 in Poland. Med Sci Monit 2020;26:e924730.

12 Cheng H-Y, Huang AS-E. Proactive and blended approach for COVID-19 control in Taiwan. Biochem Biophys Res Commun 2021;538:238-43.

13 Boletín Oficial de la República Argentina. Legislación Y Avisos Oficiales. Aislamiento social, Preventivo Y Obligatorio. Available: https://www.boletinoficial.gob.ar/detalleAviso/primera/227042/ 20200320 [Accessed Sep 2021].

14 Boletín Oficial de la República Argentina. Legislación Y Avisos Oficiales. Aislamiento social, Preventivo Y Obligatorio Y Distanciamiento social, Preventivo Y Obligatorio, 2020. Available: https://www.boletinoficial.gob.ar/detalleAviso/primera/237844/ 20201130 [Accessed Sep 2021].

15 Instituto Nacional de Estadística Y Censos de la República Argentina (INDEC). Available: https://www.indec.gob.ar/indec/web/Nivel4CensoProvincia-3-999-06-735-2010 [Accessed 14 Nov 2020].

16 Crudo F, Piorno P, Krupitzki H, et al. How to implement the framework for the elimination of mother-to-child transmission of HIV, syphilis, hepatitis B and Chagas (EMTCT plus) in a disperse rural population from the Gran Chaco region: a tailor-made program focused on pregnant women. PLoS Negl Trop Dis 2020;14:e008078.

17 Centers for Disease Control and Prevention. Interim guidelines for collecting and handling of clinical specimens for COVID-19 testing, 2021. Available: https://www.cdc.gov/coronavirus/2019-ncov/lab/ guidelines-clinical-specimens.html

18 Ministerio Nacional de Salud. Available: https://www.argentina.gob. ar/salud/coronavirus-COVID-19/definicion-de-caso [Accessed 14 Nov 2020].

19 CDC/DDID/NCIRD/Division of Viral Diseases. Cdc 2019-novel coronavirus (2019-nCoV) real-time RT-PCR diagnostic panel for emergency use only Instructions for use. CDC, Atlanta. CDC006-00019, revision: 07. effective: 07/21/2021. Available: https:// www.fda.gov/media/134922/download [Accessed Sep 2021].

20 Sigmind, 2020. Available: https://www.sigmind.net/

21 National Institutes of Health. Clinical spectrum of SARS-CoV-2 infection, 2021. Available: https://www.covid19treatmentguidelines. nih.gov/overview/clinical-spectrum/ [Accessed Sep 2021]

22 Ministerio de Salud. Gobierno de la Provincia de Buenos Aires. Available: https://www.gba.gob.ar/saludprovincia/boletin epidemiologico/semana_48_221120_al_28112020 [Accessed 20 Dec 2020].

23 Riccardo F, Ajelli M, Andrianou XD, et al. Epidemiological characteristics of COVID-19 cases and estimates of the reproductive numbers 1 month into the epidemic, Italy, 28 January to 31 March 2020. Euro Surveill 2020;25:2000790.

24 Chen N, Zhou M, Dong X, et al. Epidemiological and clinical characteristics of 99 cases of 2019 novel coronavirus pneumonia in Wuhan, China: a descriptive study. Lancet 2020;395:507-13.

25 Han Q, Lin Q, Jin S, et al. Coronavirus 2019-nCoV: a brief perspective from the front line. $J$ Infect 2020;80:373-7.

26 Buitrago-Garcia D, Egli-Gany D, Counotte MJ, et al. Occurrence and transmission potential of asymptomatic and presymptomatic SARSCoV-2 infections: a living systematic review and meta-analysis. PLOS Med 2020;17:e1003346.

27 Tsang NNY, So HC, Ng KY, et al. Diagnostic performance of different sampling approaches for SARS-CoV-2 RT-PCR testing: a systematic review and meta-analysis. Lancet Infect Dis 2021;21:1233-45.

28 Interim guidance for use of pooling procedures in SARS-CoV-2 diagnostic and screening testing, 2021. Available: https://www.cdc. gov/coronavirus/2019-ncov/lab/pooling-procedures.html [Accessed Sep 2021].

29 Pooling and serial testing Amendment for certain molecular diagnostic tests for SARS-CoV-2, 2020. Available: https://www. fda.gov/medical-devices/coronavirus-disease-2019-covid-19emergency-use-authorizations-medical-devices/in-vitro-diagnosticseuas-molecular-diagnostic-tests-sars-cov-2\#amendment [Accessed Sep 2021].

30 QGis. QGis 2020. Available: https://qgis.org/es/site/

31 Instituto Geográfico Militar. Available: https://www.ign.gob.ar/ NuestrasActividades/InformacionGeoespacial/CapassiG [Accessed Jul 2020] 